\title{
Zusammenhänge zwischen Verkehrs- und Siedlungs- entwicklung in der Schweiz seit dem Mittelalter
}

\section{Einleitung}

Die technische Entwicklung hat seit dem Mittelalter den Raum sprunghaft schrumpfen lassen, indem der Aktionsradius des einzelnen sowie der privaten und öffentlichen Institutionen ständig größer wurde. Die verschiedenen Verkehrsarten, abhängig von den Verkehrswegen und den Verkehrsmitteln, haben die raum-zeitlichen Verhältnisse sehr unterschiedlich verändert. Die ältesten und die jüngsten Kommunikationsformen - zu Fuß, auf Pferderükken oder Ochsenkarren bzw. die modernen drahtlosen Kommunikationsmittel - erschließen den Raum flächenhaft. Straßen- und weitgehend auch der Schiffsverkehr und die kabelgebundenen Informationstechnologien fördern lineare Strukturen. Mit der Eisenbahn und mit dem Flugzeug schließlich werden nur einzelne Standorte, d. h. punktuelle Raumelemente, verändert oder beeinflußt; auch die Autobahnen, obschon lineare Elemente, haben wie die Eisenbahn im Prinzip nur punktuelle Auswirkungen. Diesen von den Verkehrsmitteln her grundsätzlich verschiedenen Raumerschließungsfunktionen stehen ganz unterschiedliche Raumwiderstände gegenüber: einerseits das stark differenzierte Relief, die unterschiedlichen Bodenverhältnisse (Sumpfgebiete, Überschwemmungszonen usw.), Flüsse und Seen, andererseits erzeugen die Siedlungen selbst neue Verkehrswiderstände. Mit technischen Anlagen, insbesondere mit Verkehrsanlagen wie Brücken usw., werden Widerstände abgebaut oder zumindest reduziert.

Für die Entwicklung der Siedlungsstruktur spielen neben den lokalen absoluten, morphologischen und geoökologischen Lagefaktoren vor allem die funktionalen Lagemerkmale, wie die Wirtschaftslage, die Verkehrslage, die Ausdehnung der funktionalen Verflechtung u. a., eine wichtige Rolle. Für die Darstellung des Zusammenhanges zwischen der Siedlungs- und Verkehrsstruktur wird deshalb die Erreichbarkeit und nicht die absolute Distanz zum zentralen Faktor.Die Bedeutung der einzelnen Siedlung innerhalb dieses Systems ist ebenfalls relativ, weil sie sehr oft durch die Entwicklung der Nachbarsiedlungen auf- oder abgewertet wird, ohne daß sich die Siedlung selbst verändert. Manchmal wurden bewußt Ergänzungs- oder Konkurrenzsiedlungen gegründet oder gefördert, die sich dann gegen- seitig oder einseitig förderten oder hemmten. Vielfach änderte sich dieses Verhältnis sogar im Laufe der Zeit, wie dies bei Bern und Freiburg oder bei Biel und Nidau gezeigt werden kann.

Im folgenden versuchen wir, skizzenhaft und in vielen Ansätzen wegen fehlender Daten nur hypothetisch, für vier Phasen die räumlichen Beziehungen zwischen Siedlung und Verkehr darzustellen: Die Phase der Naturwege und des Schiffsverkehrs (um 1500), der ersten Kunststraßen oder Chausseen (um 1750-1800), des ersten Ausbaues des Schienenverkehrs als Transit- und Regionalnetz (1850-1900) sowie der Netze von Autobahn und Bahn 2000/ Alpentransit.

\section{Verkehrs- und Siedlungsstruktur um 1500}

Die Dominanz der Landwirtschaft und der vorwiegend lokalen Versorgung mit den Gütern waren gleichzeitig Voraussetzung und Ergebnis der geringen Reichweite. Die Mobilität, ausgedrückt als Anzahl Ortsveränderungen pro Tag, dürfte bei den meisten Menschen im Mittelalter kaum geringer gewesen sein als heute, nämlich mindestens zwei Ortsveränderungen pro Halbtag, meistens der Weg in die Flur oder auf die Weide, zur Kirche oder einmal wöchentlich auf den Markt und zurück. Zu Fuß oder mit dem Ochsenkarren betrug die Tagesleistung aber in der Regel nur 20-40 Kilometer und damit der Aktionsradius des einzelnen nur 10-20 Kilometer, sofern er am gleichen Tag wieder zurückkehren wollte (OHLER 1986: 141).

Für den überregionalen Verkehr bestimmten vom frühen bis ins hohe Mittelalter die Wasserstraßen und die ihnen weitgehend folgenden Landverbindungen den Verlauf der Verkehrsrouten. Sie verbanden auch die rund 15 alten Städte, die sich seit römischer Zeit erhalten hatten oder seither als Bischofssitze neu entstanden waren (GLAUSER 1987: 13).

Im Hoch- und Spätmittelalter gründeten die geistlichen und weltlichen Territorialherren in einer auBerordentlichen Gründungsphase - vorwiegend im 13. Jahrhundert - eine große Zahl weiterer Städte,

Prof. Dr. K. Aerni und Dr. Hans-Rudolf Egli, Geographisches Institut der Universität Bern, Hallerstraße 12, CH-3012 Bern 


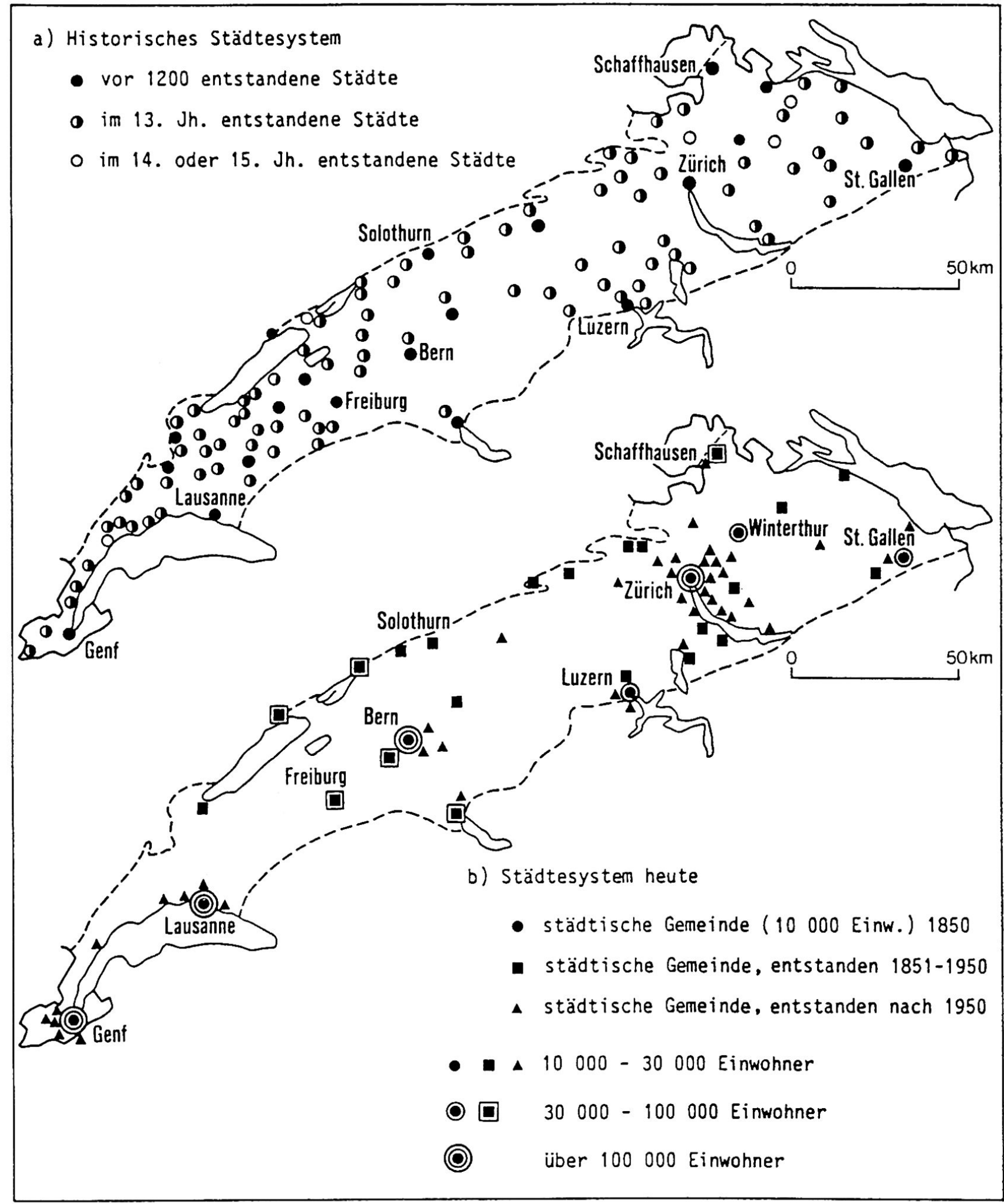

Abb. 1 Das historische und das heutige Städtesystem im schweizerischen Mittelland.

Während die historischen Städte im schweizerischen Mittelland stark dezentralisiert waren, sind die heutigen Städte (Gemeinden mit über 10000 Einwohner) in den Verdichtungsregionen Genf, Lausanne, Bern und Zürich konzentriert. Gesamthaft hat eine Verlagerung von der agrarischen Kernregion der Westschweiz in die industrielle Kernregion der Ostschweiz stattgefunden (AMMANN/SCHIB, 1958; Statistische Jahrbücher der Schweiz; in: AERNI/EGLI/FLÜCKIGER 1988: Abb. 2). 
so daß deren Zahl um 1400 rund 200 betrug (Abb. 1). Diese Klein- und Zwergstädte, von denen etliche im Spätmittelalter bereits wieder verschwanden oder sich nur noch als Dörfer weiterentwickelten (FLÜCKIGER 1984), hatten vorwiegend lokale und regionale Bedeutung. Trotzdem war damit eine Umstrukturierung des Verkehrsnetzes verbunden, das sich zunehmend von den Wasserwegen löste und die Landrouten bevorzugte.

Wichtiger Impuls für den Transitverkehr dieser Jahrhunderte war der seit den Kreuzzügen steigende Warenimport aus Asien, der von den Seestädten Italiens (Venedig, Genua) an den Alpensüdrand gelangte und von hier über verschiedene Paßrouten ins Gebiet der Champagne und des Niederrheins gelenkt wurde. Der Transitverkehr und die mittelalterliche Bedeutung Italiens für das «Deutsche Reich römischer Nation» hatte im Laufe des Spätmittelalters dazu geführt, daß die damaligen Feudalherren und die späteren eidgenössischen Orte eine aktive Paßpolitik betrieben und sich gegenseitig konkurrenzierten (AERNI 1986).

Am Ende des Hochmittelalters war die Besiedlungs- und die Rodungsphase im schweizerischen Mittelland und im Berggebiet im wesentlichen abgeschlossen; damit hatten auch die Fluren und die Weidegebiete ihre maximale Ausdehnung erreicht. Nach 1300 sind im Gebiet der heutigen Schweiz nur noch acht Städte gegründet worden. Bis heute hat demnach vorwiegend ein Siedlungsumbau als Folge eines Konzentrations- und Entleerungsprozesses und ein gewaltiger Intensivierungsproze $\beta$ stattgefunden, der zu einer völlig veränderten Siedlungsstruktur führte. Diese hat einerseits die Entwicklung der Verkehrsachsen ganz wesentlich beeinflußt, wurde aber andererseits sehr stark durch die Verkehrsentwicklung bedingt.

Die ländlichen Siedlungen mitsamt den zahlreichen Kleinstädten und das lokale Wegnetz waren fast ausschließlich durch die agrarische Landnutzung geprägt. Die Siedlungslage war durch die verfügbaren Ressourcen - Ackerland, Weide, Wasser, Bau- und Brennmaterial - bestimmt. Dabei spielte die verfügbare Menge, die Häufigkeit der Nutzung und die Transportmöglichkeit der Ressourcen und Produkte eine wesentliche Rolle. So dürfte für die im Frühmittelalter gegründeten Siedlungen das Wasser der entscheidende Standortfaktor gewesen sein, da weder Brunnen gebohrt noch Wasserleitungen gebaut werden konnten (BOELCKE 1975: 15). Später dürfte es vor allem der als Ackerland und als Weide nutzbare Boden gewesen sein, der die Ausdehnung der Flur bestimmte und damit auch die Größe der landwirtschaftlichen Siedlung. Da einerseits die Produktion standortgebunden war und andererseits ein wesentlicher Teil davon als Saatgut gelagert oder auf dem Hofe selbst verzehrt wurde, entwickelten sich die Siedlungen sehr dezentral. Die Fußwege als Flurwege, Viehwege, Kirchwege und die Karrwege als Mühlwege, Marktwege u. a. waren die weitaus häufigsten Verkehrsinfrastrukturen.

Das überregionale Verkehrsnetz der Schweiz war am Ende des Mittelalters geprägt durch zwei Hauptachsen. Einerseits war es die annähernd in west-östlicher Richtung verlaufende Verkehrsader vom Bodensee zum Genfersee, wobei die Talungen von Rhein und Aare, die Leitlinie der Juraseen und des Genfersees benutzt wurden. Der Jura und die Hügelgebiete des Alpenvorlandes grenzten den Durchgangsraum ein. Andererseits bestand ein Nord-SüdVerkehr, der sich nach Überwindung des Jura auf die drei Hauptdurchgänge der nördlichen Alpenkette ausrichtete, auf die Quertäler von Rhone und Rhein und die Reußlinie zum Gotthard. Im weiteren bestanden regionale und lokale Verkehrsnetze, ausgerichtet auf die Zentren unterschiedlicher Größe (AERNI 1990).

Die überregionalen und teilweise auch die regionalen Verkehrsnetze bezogen nach Möglichkeit Seen und Flüsse (vorwiegend talwärts) ein, so daß ein gemischtes oder "gebrochenes» Verkehrssystem bestand. Während auf den unbefestigten Landrouten im Mittelland vom Säumen auf Tragtieren allmählich auf Karren und Wagen umgestellt wurde, mußte der transalpine Verkehr auf der Stufe des Saumverkehrs verbleiben. Ein illustratives Beispiel für dieses «gebrochene» Verkehrssystem ist die Regelung des Warenverkehrs von Thun über die Grimsel. Ein Schiffahrtsreglement galt für die Strecke ThunBrienz, ein Reglement für den Landkarrer ordnete den Transport von Brienz nach Meiringen, und Säumerreglemente umschrieben schließlich den Transport über Grimsel und Gries ins Pomat.

Handelshindernisse waren auf all diesen Wegen nicht bloß die geländebedingten Erschwernisse, sondern auch die zahlreichen Zölle und die lokalen Transportmonopole der Säumer.

\section{Verkehrs- und Siedlungsstruktur in der zweiten Hälfte des 18. Jahrhunderts}

Die im Spätmittelalter ausgeformte Eidgenossenschaft setzte sich aus Stadt- und Länderorten zusammen. Während die Stadtorte als Erben mittelalterlicher Territorialherren ihren Einflußbereich durch die Entwicklung absolutistischer Herrschaftsansprüche zu Untertanengebieten umgestalteten, behielten die Länderorte demokratische Strukturen. Damit wurde die weitere Gründung von Städten unterbunden. Als Folge des Bevölkerungswachstums und des wachsenden wirtschaftlichen Austausches entwickelten sich neue Marktorte als regionale Zentren. Den rund 200 Städten und 30 Märkten der Zeit um 1400 standen am Ende des 18. Jahrhunderts nach wie vor etwa 200 Städte unterschiedlichster Größe gegenüber, die Zahl der Marktorte aber war nach PEYER (1979: 22) auf rund 300 gestiegen. 
Die regionale Entwicklung führte zu steigenden Warenströmen im Binnenverkehr. Der Transitverkehr stieg jedoch nicht im gleichen Ausmaß an, da als Folge der seit dem 14. Jahrhundert erfolgten Entdeckung neuer Seewege das Mittelmeer seine frühere Transitfunktion verloren hatte und der Handelsraum Europa seine atlantische Seite (Niederlande, England) entwickelt hatte.

Eine ausgeprägte Verkehrspolitik ging in der nachmaligen Schweiz vom 17. Jahrhundert an nur von wenigen Stadtorten und von den an Transitrouten liegenden Länderorten aus.

Die frühe Neuzeit hat das mittelalterliche Verkehrsnetz der unbefestigten Naturstraßen übernommen und es nur allmählich verbessert. Die wichtigste Verbindung war die alte West-Ost-Verbindung aus der Waadt über Murten-Aarberg-Solothurn-Aargau mit der Abzweigung über den Hauenstein nach Basel. Im 18. Jahrhundert suchte Bern durch seinen Straßenbau den Transit von Murten über Bern Richtung Aargau zu lenken, um damit das Hoheitsgebiet von Solothurn umgehen zu können. Daneben behielt auch die Wasserlinie mit den Juraseen und der Aare ihre Bedeutung.

Im Nord-Süd-Verkehr dominierten in der Verkehrsspanne zwischen Basel/Süddeutschland und Mailand die Bündnerpässe und der Gotthard (HÄRRY 1911: 149). Im Westen wurde der Simplon unter dem tatkräftigen Kaspar Jodok von Stockalper (1609-1691) im späteren 17. Jahrhundert zur bedeutendsten Verbindung zwischen Lyon und Mailand, die jedoch nach seinem Tode wieder zerfiel.

Verkehrsmittel und Straßen im Mittelland begannen sich gegenseitig zu beeinflussen. Die Transportarten stiegen vom Saumtier (mit rund $150 \mathrm{~kg}$ Traglast) über den zweirädrigen Karren $(300-400 \mathrm{~kg}$ ) zum vierrädrigen Wagen $(800 \mathrm{~kg}$ ) bis zu den Frachtwagen mit rund $2500 \mathrm{~kg}$ Kapazität (GLAUSER 1987: 12). Diese Entwicklung erforderte eine Verbesserung des Straßenkörpers und eine entsprechende Entwicklung der Hufbeschläge, um die Zugtiere beim Übergang von den unbefestigten Naturstraßen zu den Hartstraßen zu schützen.

Hinter diesen technologischen Neuerungen stand eine mehr oder weniger bewußte Verkehrspolitik der Territorialherrschaften. Im Zeitalter des Merkantilismus erkannten die absolutistischen Staaten den Zusammenhang zwischen Straßenqualität, Verkehrsmenge, Zollertrag und Staatsfinanzen und begannen planmäßig mit dem Bau von Kunststraßen. Wegweisend in Europa waren Frankreich mit seinen «Chausseen» und Bern mit dem 1742 einsetzenden Bau eines sternförmig auf die Hauptstadt ausgerichteten Straßennetzes (Grosjean 1973: 40). Damit wurde in der Schweiz eine Abkehr vom alten Prinzip des auf den Anstößern und Gemeinden lastenden Straßenunterhaltes zugunsten einer staatlichen Verkehrspolitik eingeleitet (KAISER 1986).
Die Auswirkungen der neuen Technik waren beträchtlich. Bern gelang im 17./18. Jahrhundert nicht nur die Verbesserung des Straßennetzes, sondern es verstand auch, dessen Nutzung durch das von der Familie Fischer aufgebaute Postnetz zu optimieren (RYHINER/KLÖTI 1990: 428).

Die Fortschritte in der Raumüberwindung im Mittelland zeigt sich beispielsweise daran, daß 1780 die Strecke Genf-Lausanne auf der Chaussee in 11 Stunden zurückgelegt werden konnte, die Strecke Genf-Neuenburg auf unbefestigten Straßen dagegen drei Tage erforderte (HÄRRY 1911: 149 f.). Die Fahrgeschwindigkeit auf Naturstraßen entsprach dem Schrittempo, die Postwagen auf den KunststraBen dagegen konnten mit etwa 8,5 Stundenkilometern fahren. Damit begann im Mittelland eine Verlagerung des Transitverkehrs auf die ausgebauten Straßen. Über die Alpenpässe dagegen war noch kein Fahrverkehr möglich, so daß noch keine Verkehrskonzentration auf einzelne Linien stattfand (vgl. Tab. 1). Dies änderte sich erst im 19. Jahrhundert mit dem Bau von Fahrstraßen (1805 Simplon, San Bernardino und Splügen 1823, HauensteinGotthard 1819-1832).

Tab. 1 Transportzeiten Basel-Turin 1804 und 1989 über vier Alpenpässe in Stunden

\begin{tabular}{|ccccc|}
\hline & $\begin{array}{c}\text { Großer } \\
\text { St. Bernhard }\end{array}$ & Simplon Gotthard & $\begin{array}{c}\text { Bündnerpässe } \\
\text { (San Bernardino) }\end{array}$ \\
\hline 1804 & $82^{\star}$ & $102^{\star}$ & $96^{\star}$ & $101^{\star}$ \\
1989 & $10-12$ & $10-12$ & $9-10$ & 15 \\
\hline
\end{tabular}

* Durchschnittswerte

Quellen: HÄRRY 1911: 146; Angaben der Transportunion Basel, Dezember 1989

\section{Verkehrs- und Siedlungsstruktur in der 2. Hälfte des 19. Jahrhunderts}

Obschon die Schweiz zu Beginn des 19. Jahrhunderts das verhältnismäßig am stärksten industrialisierte Land des europäischen Kontinents war - nur das Wallis, das obere Tessin, größere Teile Graubündens sowie das westliche Mittelland zwischen Bern und Genf waren von der Industrialisierung kaum erfaßt (IM HOF 1980: 718) - wohnten 1850 erst knapp 200000 Menschen oder 6,4\% der Bevölkerung in Gemeinden mit über 10000 Einwohnern (GÜLLER/GROSS/MUGGLI 1981: 19), d. h. in Städten nach statistischer Definition; Zürich mit rund 42000 Einwohnern war schon damals die größte Stadt, Luzern mit knapp über 10000 die kleinste. Die starke Dezentralisierung der Industrie und der stark föderalistische Staatsaufbau, insbesondere uneinheitliche Maße und Gewichte und die kantonalen Zölle, hemmten den Eisenbahnbau und ver- 
zögerten den Konzentrationsprozeß. Bestand 1850 in Großbritannien bereits ein $10600 \mathrm{~km}$ langes und in Deutschland ein $6000 \mathrm{~km}$ langes Streckennetz, so waren in der Schweiz erst $27 \mathrm{~km}$ gebaut (DTVBrockhaus-Lexikon, Bd. 4: 324). Nach anfänglichem Schwanken in den jungen eidgenössischen Räten wurde 1852 für den privaten Bahnbau entschieden, was dem wirtschaftlichen Liberalismus und dem damaligen Zeitgeist entsprach; der Bund begnügte sich im Eisenbahngesetz mit einem Aufsichtsrecht. Der Bahnbau erfolgte damit nicht nach staatspolitischen, geopolitischen und volkswirtschaftlichen Gesichtspunkten, sondern rein nach betriebswirtschaftlichen. Es entstand bis um 1870 ein Eisenbahnnetz mit einer Hauptlinie von Rorschach nach Genf - zwischen Herzogenbuchsee und Lausanne war sie mit einer Linie über Bern-Freiburg und einer zweiten Linie dem Jurasüdfuß entlang doppelt geführt - und mit Stichlinien von Olten nach Basel und Luzern, von Zürich nach Chur, von Bern nach Thun und von Lausanne nach Sierre. Dieses Netz diente in erster Linie der Verteilung von Steinkohle und Getreide. Einzelne Kantone beteiligten sich an den Bahngesellschaften und korrigierten die Linienführung nach regionalen $\mathrm{Ge}-$ sichtspunkten. Bis 1860 waren bereits über 1000 Streckenkilometer gebaut, im Jahre 1880 waren es $2500 \mathrm{~km}$. Nachdem 1869 durch die Eröffnung des Suezkanals die Mittelmeerhäfen wiederum große Bedeutung erlangt hatten, wurde im selben Jahr der Gotthardbahnvertrag mit Italien und dem Norddeutschen Bund abgeschlossen. Mit dem Eisenbahngesetz von 1872 wurde der Bund ermächtigt, selber Bahnen zu bauen und zu betreiben und Bahnen zurückzukaufen. Der Rückkauf erfolgte allerdings erst in den Jahren 1901-1903, der Betrieb der Schweizerischen Bundesbahnen begann am 1. Januar 1901 (GROSJEAN 1978: 60). Zu diesem Zeitpunkt wohnten bereits $22 \%$ der Schweizer Bevölkerung in städtischen Gemeinden. Die starke räumliche Ausdehnung der Städte machte dann aber den Aufbau innerstädtischer Verkehrsnetze nötig, um die lokale Erschließung und damit die Erreichbarkeit zu verbessern. Genf erhielt 1862 als erste Schweizer Stadt eine von Pferden gezogene StraBenbahn. 1877 wurden in Genf und Lausanne die ersten Dampfstraßenbahnen eingeführt, zwischen 1894 und 1898 entstanden dann in allen größeren Städten elektrische Straßenbahnen (ASSEO 1990: 187).

Mit der Eisenbahn wurden einerseits die Städte punktuell erschlossen und ihre Erreichbarkeit untereinander, nicht aber aus ihrem Umland, ganz wesentlich verbessert. So verkürzte sich die Reisedauer von Bern nach Zürich von rund 13 Stunden mit der Postkutsche auf $41 / 2-51 / 2$ Stunden mit der 1858 durchgehend benützbaren Bahn. Im NordSüd-Verkehr reduzierte sich mit dem Bau des Gotthard-Eisenbahntunnels die Reisezeit von Luzern nach Bellinzona sogar von $161 / 2$ Stunden auf 41/2-71/2 Stunden, die Zahl der Reisenden betrug im ersten Betriebsjahr 1883 mit rund 250000 bereits mehr als das Dreifache gegenüber den letzten Betriebsjahren der Postkutschen.

Andererseits hatte der Bahnbau im Mittelland billige Getreideimporte zur Folge, die die Landwirtschaft derart schwächten, daß es in den 1870er Jahren zu einer gravierenden Agrarkrise kam (EGLI 1990a: 369 f.). Die Auswanderung ins Ausland und die Abwanderung in die Städte verstärkten die Bevölkerungskonzentrationen und damit die Verstädterung in sehr hohem Maße (EGLI 1986). Diesem Konzentrationsprozeß lief die touristische Entwicklung im Alpenraum entgegen - vor dem 1. Weltkrieg vorwiegend im Berner Oberland und im Kanton Graubünden. Auch für diese Frühphase des Fremdenverkehrs spielte die EisenbahnerschlieBung eine zentrale Rolle (EGLI 1990b: $60 \mathrm{ff}$.).

Zusammenfassend ist der Eisenbahnbau die bedeutendste Ursache der Ausbildung der heutigen Siedlungsstruktur und Verteilung der Wohnbevölkerung und der Arbeitsplätze, da die Eisenbahn durch die punktuelle Erschließung des Raumes auf nationaler, regionaler und lokaler Ebene einen außerordentlichen Konzentrationsproze $\beta$ auslöste und förderte (s. dazu auch: GROSJEAN 1984).

\section{Verkehrs- und Siedlungsstruktur der Gegenwart}

Der Verkehr als Gesamterscheinung gliedert sich in unserem Jahrhundert zunehmend auf und trennt sich räumlich auf spezifische Transportnetze. Der Nachrichtenverkehr benützt in steigendem Maße neben der traditionellen Post kabelgebundene und drahtlose Verbindungen, wodurch sich im Nachrichtenwesen der Raum aufhebt. Der Güter- und der Personenverkehr beginnen sich auf spezialisierte Verbindungen abzustützen, am deutlichsten im modernen Schiff- und Bahnverkehr. Hinter dieser Entwicklung stehen als Auslöser die durch die technische Entwicklung möglich gewordene individuelle Mobilität und die wachsende räumliche Spezialisierung der Wirtschaft, verbunden mit neuen wirtschaftspolitischen Zusammenschlüssen. In diesem Zusammenhang ist von Bedeutung, daß die Alpenstaaten Schweiz und Österreich der EG nicht angehören und damit der Alpenraum verkehrspolitisch als Sperriegel Italien vom wirtschaftlichen Schwergewichtsraum der EG trennt.

Die Schweiz hat seit 1950 das Nationalstraßennetz weitgehend verwirklicht und ist im Begriff, nun auch mit den Projekten «Bahn 2000» und «Alpentransit» den Schienenverkehr zu erneuern.

Das Autobahnnetz hat für den Straßenverkehr neue Zeitverhältnisse geschaffen (Abb.2). Es entstand aus der Notwendigkeit, daß das bisherige Straßennetz der seit 1950 wachsenden Verkehrswelle 
(152 000 Personenwagen 1950, 3 Mio. 1990) nicht mehr gewachsen war. 1960 genehmigte das Parlament ein Bauprogramm für $1830 \mathrm{~km}$ NationalstraBen. Das Konzept beruhte ursprünglich auf der Verbindung der fünf Großstädte Genf, Lausanne, Bern, Basel und Zürich unter Einbezug der Mittelzentren. Für den transalpinen Verkehr wurde 1964 auf private Initiative hin der Scheiteltunnel durch den Großen St. Bernhard eröffnet, danach folgten die Nationalstraßentunnel San Bernardino (1967) und Gotthard (1980) sowie die Straße über den Simplon. Mit dem Bau von Scheiteltunneln wurden im transalpinen Verkehr die Höhendifferenzen und Witterungseinflüsse so reduziert, daß die Straßenübergänge ganzjährig und in wenigen Stunden befahren werden konnten.

Diese Verbesserungen des Straßennetzes waren ursprünglich für den Personenverkehr vorgesehen, wurden aber sehr rasch auch vom Güterverkehr übernommen und von Dienstleistungs- und Verteilzentren ausgenützt. Daß damit im Güterverkehr eine entscheidende Weichenstellung zugunsten der Straße und in Abkehr vom bisherigen Bahntransport vorgenommen wurde, erkannte man damals nicht. Die Eröffnung der Straßentunnel Mont Blanc (1965) und Fréjus (1980) sowie die Autobahn über den Brenner (1959-72) verschärften die Entwicklung auf europäischer Ebene (Abb. 3). Die daraus entstandenen Folgen für Mensch und Umwelt sind bekannt.

Nachdem die öffentliche Hand in der Nachkriegszeit einseitig den Straßenverkehr gefördert hatte, setzte nach 1970 eine Neubewertung des Schienenverkehrs (und allgemein des öffentlichen Verkehrs) ein. 1972 wurde in der «Gesamtverkehrskonzeption» vom Bund eine neue «Eisenbahn-Haupttransversale» NHT zwischen Genfersee und Bodensee vorgeschlagen. Gleichzeitig erhielten die SBB den Auftrag, ein Projekt für eine neue Alpentransversale vorzubereiten. Dies wäre im Hinblick auf die steigende wirtschaftliche Zusammenarbeit der Europäischen Gemeinschaft (EG) der richtige Zeitpunkt gewesen, um den Anschluß an das künftige europäische Schnellbahnnetz zu finden.

Der Konjunktureinbruch von 1974 verzögerte jedoch das Projekt einer Alpentransversale für die Bahn. Für das Mittelland dagegen wurde 1985 das Schnellbahnkonzept der NHT, welches vor allem

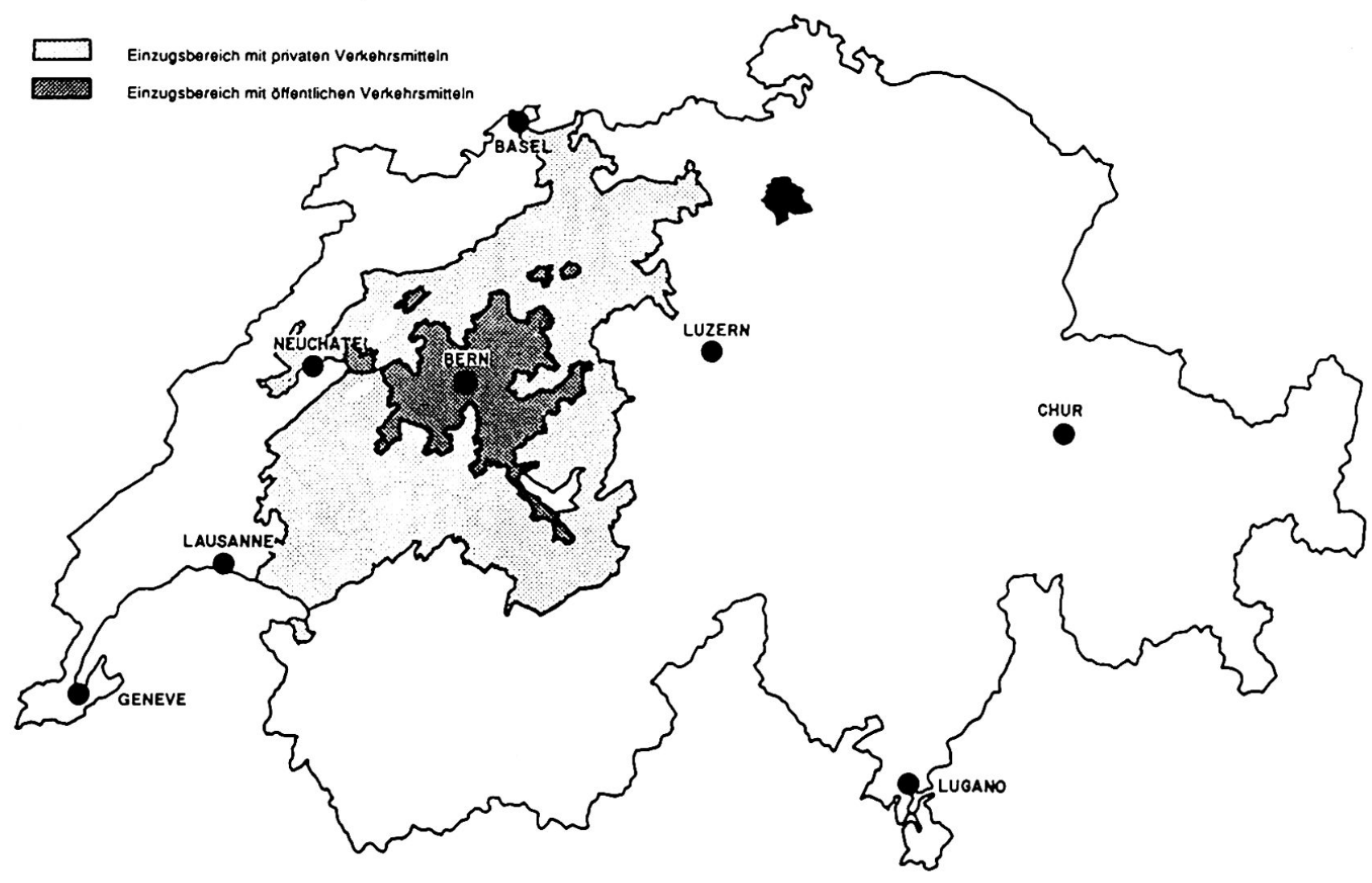

Abb. 2 Erreichbarkeit des Umlandes von Bern in 60 Minuten Reisezeit mit privaten und öffentlichen Verkehrsmitteln 1984. Das Netz dses öffentlichen Verkehrs (ÖV-Netz) berücksichtigt alle Bahnverbindungen, Postautokurse und die regionalen Bahnlinien. Die ÖV-Reisezeiten enthalten Anmarschzeit zu den Haltestellen (5-10 Min.), Wartezeit (5-12 Min.), Fahrzeit inklusive Umsteigezeit und Abmarschzeit (5-10 Min.). Im Netz des privaten Verkehrs (PV-Netz) sind alle National- und Hauptstraßen sowie die wichtigsten Nebenstraßen enthalten. Die PV-Reisezeit setzt sich aus dem Fußweg zum Auto und der Fahrt zum übergeordneten Netz (3-10 Min.), der Fahrzeit auf dem übergeordneten Netz und dem Zeitaufwand vom Netz zum Parkplatz und von dort zum Ziel zusammen (3-10 Min.) (WÜEST 1987: 3 f. und 24). 
die Zentren begünstigt hätte, durch das den regionalen Bedürfnissen angepaßtere Konzept «Bahn 2000» ersetzt (BR 1985).

Inzwischen hat der umweltbelastende Straßenverkehr allgemein und der vom Wachsen der EG induzierte Straßentransit weiterhin zugenommen. Die Schweiz reagierte wirksam durch die Gewichtsbeschränkung für Lastwagen auf 28 Tonnen, die Erhebung einer Pauschalabgabe auf dem Schwerverkehr und durch Verkehrsbeschränkungen (Fahrverbot an Feiertagen und in der Nacht) für den transalpinen Straßengüterverkehr.

Die EG fordert nun seit einiger Zeit unmißverständlich die Zulassung der 40-Tonnen-Lastwagen oder mindestens die Öffnung eines Transitkorri- dors. Zur Zeit laufen die Vorbereitungen für die «Alpen-Transit» (BR 1990), welche eine Netzlösung unter Einbezug von Lötschberg und Gotthard vorsieht. Beide Projekte sollen zudem miteinander verbunden werden. Gleichzeitig aber wird als Sofortmaßnahme die Gotthardbahn für den «kombinierten Verkehr" hergerichtet, und analoge Anpassungen an der Lötschberglinie sind vorgesehen. Die Kosten für die «Bahn 2000» werden heute auf 9 Mia. Franken geschätzt, jene der «Alpen-Transit» auf 24 Mia. Franken - die Einnahmen des Bundes betrugen 1988 rund 28 Mia. Franken, die Schulden 26 Mia. Franken (BFS 1991: 362 f.).

Für die künftige Verkehrsstruktur der Schweiz ist durch die Realisierung der heutigen Bahnprojekte

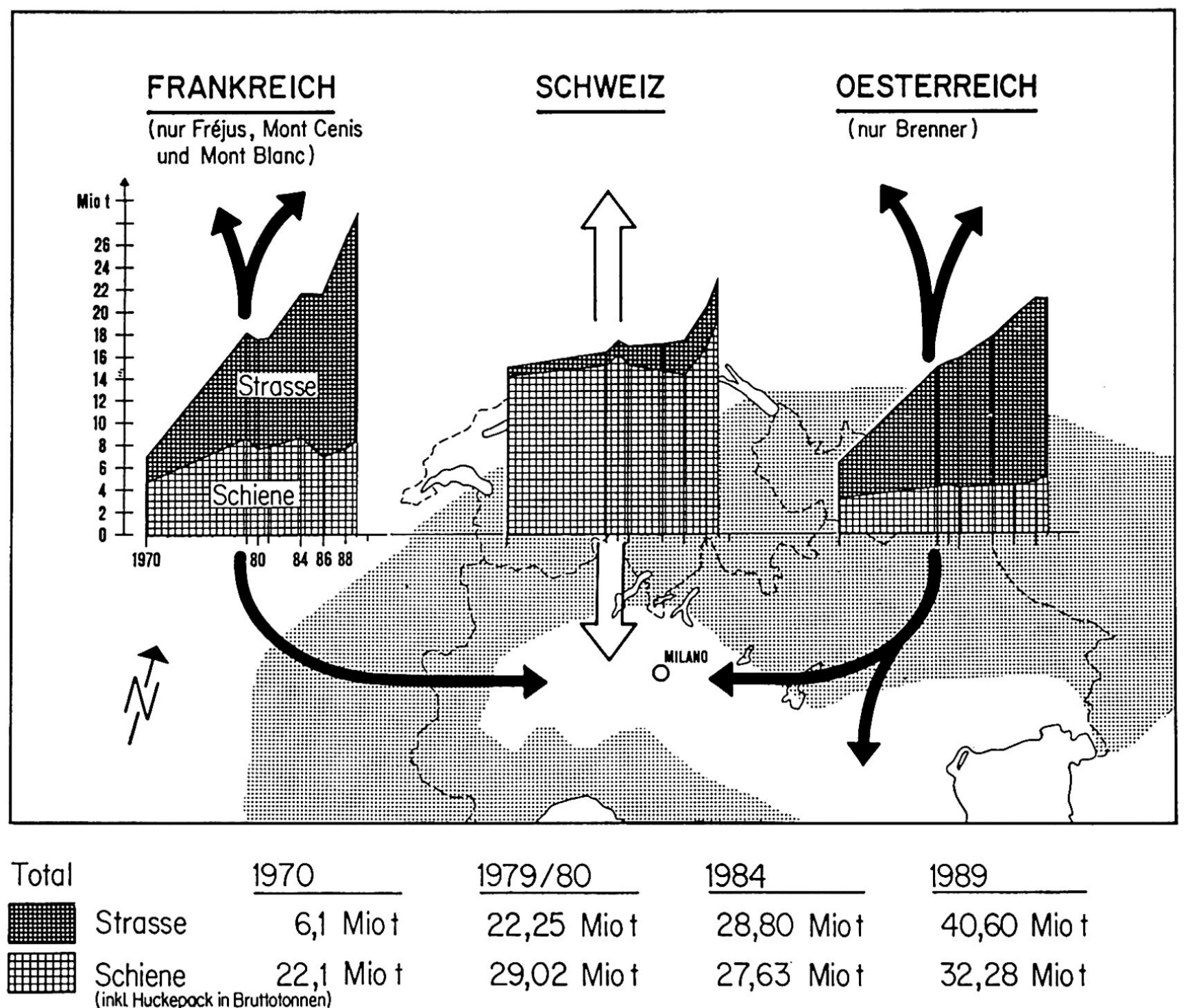

(Quelle: EVED, Dienst GVF)

Entwurf: K. Aerni, U. Seewer 1991

Abb. 3 Der transalpine Güterverkehr zwischen Italien und Nordeuropa von 1970-1989, gegliedert nach Alpensegmenten und Verkehrsträgern in Millionen Tonnen (inklusive Huckepackverkehr auf der Schiene).

Deutlich erkennbar ist die geringe Zunahme des Bahnverkehrs im Beobachtungszeitraum und das rasche Wachstum des Straßenverkehrs außerhalb der Schweiz (EVED, GVF-Bericht 2/91: Anhang 1, Tab. 1; GVF-News Nr. 12/1991). 
die Vision einer «Stadt Schweiz» erkennbar, deren Zentren durch Intercityzüge im Halbstundentakt verbunden sind und die mit ausländischen Zentren nach der Realisierung des europäischen Hochgeschwindigkeitsnetzes in wenigen Stunden verbunden ist.

\section{Zusammenfassung}

Die Wechselwirkungen zwischen Verkehr und Siedlung in der Schweiz seit 1500 zeigen, daß die verschiedenen technischen Innovationen im Straßenund Bahnnetz die Raum-Zeit-Verhältnisse und damit die Erreichbarkeit regional unterschiedlich veränderten. Der Alpenraum blieb wegen des reliefbedingten Verkehrswiderstandes vom Bau der Fahrstraßen an stets um eine technologische Generation im Rückstand. Im Siedlungsbereich führte der Ausbau der Verkehrsinfrastruktur zu einer immer ausgeprägteren Bevorzugung der durch sie verbundenen Zentren, damit zu einer verstärkten hierarchischen Struktur der Städte und einer fortschreitenden Raumspezialisierung, die für die Zukunft schwierige Entwicklungsprobleme für die Gestaltung des ländlichen und des städtischen Lebensraumes stellt.

\section{Literatur}

AERNI, K. (1986): Die bernische Alpenpaßpolitik vom Mittelalter bis zur Frühen Neuzeit. In: Aerni, K./Herzig, H. (Hrsg.), 1986: Historische und aktuelle Verkehrsgeographie der Schweiz. Geographica Bernensia, Heft G 18, 57-84.

AERNI, K. (1990): Sic transit gloria alpium. In: Mayer-Tasch, P. C. et al. (Hrsg.): Das Drama der Mobilität. Zürich, 162-102.

AERNI, K. EGLI, H.-R. und FLÜCKIGER, P. (1988): Das Schweizerische Mittelland. Strukturen und Prozesse. In: Geographische Rundschau 40, S. 49-57.

ASSEO, D. (1990): Transport en commun et nouvelles technologies: le cas de Genève au XIXe siècle. In: Schweizerische Zeitschrift für Geschichte, Vol. 40, S. 185-206.

BFS - Bundesamt für Statistik (1991): Statistisches Jahrbuch der Schweiz 1990. Zürich.

BOELCKE, W. (1975): Römisches Erbe, alemannische Landnahme und die Entstehung der Grundherrschaft im deutschen Südwesten. In: Ludwigsburger Geschichtsblätter 27, 1975, S. 5-57.

BR - Schweizerischer Bundesrat (1985): Bericht über das Konzept BAHN 2000 und Borschaft über den Bau neuer Linien der Schweizerischen Bundesbahnen. Nr. 85.074. Bern.

BR - Schweizerischer Bundesrat, 1990: Botschaft über den Bau der schweizerischen Eisenbahn-Alpentransversale (Alpentransit-Beschluß), Nr. 90.040. Bern.

DTV-Brockhaus-Lexikon (1988): DTV-Brockhaus-Lexikon in 20 Bänden. Mannheim/München.

EGLI, H.-R. (1986): Ländliche Neusiedlungen in der Schweiz vom Ende des 19. Jahrhunderts bis zur Gegenwart. In: Erdkunde, Bd. 40, Heft 3, S. 197-207.
EGLI, H.-R. (1990a): Les objectifs de la politique agricole suisse. In: Nouvelle Géographie de la Suisse et des Suisses. Lausanne, S. 363-375.

EGLI, H.-R. (1990b):"Landschaft und Siedlung. In: Die Bauernhäuser des Kantons Bern, Bd. 1: Das Berner Oberland. Basel, 1990, S. 11-63.

EVED, Dienst für Gesamtverkehrsfragen (1991): Transalpiner Güterverkehr 1989. Überblick über die Entwicklung seit der Eröffnung des Gotthard-Straßentunnels 1980. (GVF-Bericht 2/91). Bern.

FLÜCKIGER, R. (1984): Mittelalterliche Gründungsstädte zwischen Freiburg und Greyerz als Beispiel einer überfüllten Städtelandschaft im Hochmittelalter. (Freiburger Geschichtsblätter, Bd. 63, 1983/84). Freiburg.

GLAUSER, F. (1987): Verkehr im Raum Luzern-Reuß-Rhein im Spätmittelalter - Verkehrsmittel und Verkehrswege. In Jb. der Hist. Ges. Luzern, H. 5, 2-19.

GROSJEAN, G. (1973): Kanton Bern - Historische Planungsgrundlagen. Planungsatlas Kanton Bern, 3. Lieferung. Bern.

GROSJEAN, G. (1978): Die Schweiz: Geopolitische Dynamik und Verkehr. (Geographica Bernensia, Bd. U 3). Bern.

GROSJEAN, G. (1984): Historische Faktoren und Bedingungen der Herausbildung des schweizerischen Siedlungs-, Flächennutzungs- und Kommunikationsnetzes. (Arbeitsbericht 48, Nationales Forschungsprogramm "Regionalprobleme in der Schweiz"). Bern.

GÜLLER, P., GROSS, T. und MUGGLI, C. (1981). Historischprospektive Reflexionen zum schweizerischen Stadtsystem. (Arbeitsbericht 22, Nationales Forschungsprogramm "Regionalprobleme in der Schweiz"). Bern.

HÄRRY, A. (1911): Die historische Entwicklung der schweizerischen Verkehrswege mit besonderer Berücksichtigung des Transits und der Fluß-Schiffahrt. Erster Teil. Nordostschweizerischer Verband für Schiffahrt Rhein-Bodensee, Verbandsschrift Nr. 12. Frauenfeld.

IM HOF, U. (1980): Ancien Régime. In: Handbuch der Schweizer Geschichte, Bd. 2. Zürich, S. 673-784.

KAISER, P. (1986): Die Straßen der Nordostschweiz im 17. und 18. Jahrhundert. Vorbemerkungen zu einer regionalen Straßengeschichte. In: Aerni, K. und Herzig, H. (Hrsg.): 1986: Historische und aktuelle Verkehrsgeographie der Schweiz. Geographica Bernensia, Heft G 18, 85-100.

MEIER, Th. (1988): Territorialisierung der Gesellschaft? (Überlegungen zu Raum und Raumstrukturen aus mediävistischer Sicht. In: DISP Nr. 92 (Dokumente und Informationen zur Schweizerischen Orts-, Regional- und Landesplanung.) Zürich, S. 29-35.

NEUHAUS, W. (1987): Bern-Aarau in drei Stunden. In: Der Bund, 8.8.1987, Nr. 103.

OHLER, N. (1986): Reisen im Mittelalter. München.

PEYER, H.C. (1979): Gewässer, Grenzen und Märkte in der Schweizergeschichte. Mitt. der Antiquarischen Gesellschaft in Zürich, Band 48, Heft 3.

RYHINER, J.F., VON, und KLÖTI, T. (1990): Die Post: Ein «Geschäft" für wen? Geschichte des bernischen Postwesens von 1648-1798 und J.F. von Ryhiners "Bericht über das Postwesen in Helvetien, 1793". Bern.

WÜEST, H. (1987): "Raum-Sensor» - Erreichbarkeitsstudie mit öffentlichen und privaten Verkehrsmitteln. Studie im Auftrag des Bundesamtes für Raumplanung. Bern.

WYSS-NIEDERER, A. (1979): Sankt Gotthard - Via Helvetica. Lausanne. 